\title{
Magnetotactic Bacteria at the Geomagnetic Equator
}

\author{
Abstract. Magnetotactic bacteria are present in fresh water and marine sediments \\ of Fortaleza, Brazil, situated close to the geomagnetic equator. Both South-seeking \\ and North-seeking bacteria are present in roughly equal numbers in the same \\ samples. This observation is consistent with the hypothesis that the vertical \\ component of the geomagnetic field selects the predominant polarity type among \\ magnetotactic bacteria in natural environments.
}

Several species of magnetotactic bacteria have been observed in aquatic sediments of the Northern and Southern Hemispheres (1-5). Each bacterium contains magnetosomes consisting of enveloped, single-domain magnetite particles. The magnetosomes are often arranged in chains with a magnetic dipole moment, parallel to the axis of motility, sufficiently large that the cell is oriented along the geomagnetic field lines as it swims $(6,7)$. Cells with North-seeking pole forward swim North along the magnetic field lines; cells with the South-seeking pole forward swim South. Because of the inclination of the geomagnetic field, North-seeking cells migrate downward in the Northern Hemisphere and upward in the Southern Hemisphere; Southseeking cells migrate downward in the Southern Hemisphere and upward in the Northern Hemisphere. Magnetotactic bacteria in Northern Hemisphere sediments are almost exclusively Northseeking (1-3), while bacteria in Southern Hemisphere sediments are almost exclusively South-seeking $(4,5)$. Thus downward directed motion is advantageous for, and upward directed motion is detrimental to, survival of these organisms; and the vertical component of the geomagnetic field selects the predominant cell polarity. If magnetotactic bacteria exist at the geomagnetic equator where the magnetic field lines are horizontal, neither polarity should be selected. We report the observation of various morphological types of magnetotactic bacteria in aquatic sediments close to the geomagnetic equator. North-seeking as well as South-seeking bacteria are present in roughly equal numbers in the same sediment samples.

Samples of fresh water and marine sediments were collected in the vicinities of Fortaleza and Rio de Janeiro, Brazil. At these locales, the total intensity of the geomagnetic field is 0.25 to 0.28 gauss (8), approximately one-half the intensity at locales in New England and New Zealand where magnetotactic bacteria have previously been found. Fortaleza is situated close to the geomagnetic equator (inclination $<4^{\circ}$ ) while at Rio de Janeiro the inclination of geomagnetic field is $25^{\circ}$ to $30^{\circ}$ South. Magnetotactic responses of bacteria in sediment samples were observed in uniform magnetic fields, up to 3 gauss, provided by a pair of Helmholtz coils mounted on either side of a Nikon SMZ-10 stereomicroscope. The magnetic field axis was aligned parallel to the horizontal component of the geomagnetic field. The direction of current flow in the coils and hence polarity of the imposed magnetic field was selected with a toggle switch. Bacteria from sediment samples collected in Fortaleza migrated along the magnetic field lines, some in the field direction (North-seeking) and roughly equal numbers in the same sample opposite to the field direction (South-seeking) When the imposed field was reversed, both groups of bacteria executed $U$ turns and swam opposite to the initial direction. Bacteria from sediment samples collected in Rio de Janeiro migrated opposite to the field direction (South-seeking) only, and also reversed direction on reversal of the field. Migration rates of bacteria from both locales were comparable to those of other magnetotactic bacteria.

The bacteria were subjected to a demagnetizing procedure by exposing them to and subsequently slowly moving them away from an alternating $60-\mathrm{Hz}$ magnetic field over 1000 gauss produced by a small hand-held magnetic tape degausser. North-seeking and South-seeking bacteria from Fortaleza were first separated by their magnetotactic response, placed in separate water drops on a microscope slide, and then exposed. Whereas before exposure all the bacteria in each drop swam exclusively in the field direction, or opposite to the field direction, after exposure each drop contained appoximately equal numbers swimming in and opposite to the field direction. Similar results, previously re- ported for other magnetotactic bacteria (4), were also obtained with South-seeking bacteria from Rio de Janeiro. Thus each bacterial magnetic dipole is essentially a single magnetic domain and cannot be demagnetized. However, the polarity can be reversed (2) and the demagnetization procedure results in the reversal of about one-half of the dipoles of the bacterial population in each drop. Preliminary electron microscope studies show that North- and South-seeking bacteria from Fortaleza are morphologically identical and contain intracytoplasmic electron opaque particles (9). Magnetosomes consisting of intracytoplasmic, enveloped, magnetite particles are a characteristic of all magnetotactic bacteria studied to date $(10-12)$.

In order to determine experimentally the effect of a vanishing vertical magnetic field, sediments samples from New England initially containing exclusively North-seeking bacteria were placed in a Mumetal enclosure in New England in which the magnetic field intensity was less than one hundredth the intensity outside the enclosure. The polarities of bacteria from both experimental and control samples placed outside the enclosure were monitored periodically over several weeks, that is, many bacterial generations. In the experimental samples, numbers of South-seeking cells increased with time until the ratio of South-seeking bacteria to North-seeking bacteria approached 1.0. No such changes occurred in the control samples. Thus, in natural habitats and in laboratory experiments neither cell polarity is selected in the absence of a vertical magnetic field. These findings complement previous observations that a predominant cell polarity is selected, depending on the sign of the vertical component of the ambient magnetic field (4). Because oxygen is toxic for these microaerophilic or anaerobic organisms (1), cells whose polarity causes them to be directed toward and kept in the sediments and away from the water surface will be favored. 
Because of the horizontal orientation of the magnetic field at the geomagnetic equator, the motion of magnetotactic bacteria there will be directed horizontally. This could be advantageous to bacteria of either polarity in reducing detrimental upward migration, compared to random motion. Extended straight-line motion could also be advantageous as an escape response, for population dispersal, and as a means of outrunning chemical diffusion and finding more suitable environments $(5,13,14)$. Their magnetism would also cause cells to localize in regions of high magnetic flux density surrounding materials with high magnetic susceptibility in the environment ( 7 , 15); and bacteria that are within about 4 $\mu \mathrm{m}$ of each other will experience magnetic interactions stronger than thermal energy. These interactions may be advantageous but would not favor either polarity at any latitude.

Since the total intensity of the geomagnetic field in Brazil is less than one-half the intensity of the field in New England, Brazilian bacteria would need larger magnetic moments on the average to maintain the same ratio of magnetic-tothermal energy and hence the same degree of alignment in water at ambient temperatures for the magnetotactic response (7). This hypothesis could be confirmed by detailed electron microscope studies of their magnetosomes or by observation of their swimming response in the magnetic field direction as a function of magnetic field strength (16).

Finally, the presence of magnetotactic bacteria at the geomagnetic equator implies their ability to survive periodic reversals or excursions of the geomagnetic field (17) even at latitudes with large inclination. Because of the nondipolar contribution to the geomagnetic field, the field intensity at any point on the earth's surface does not completely vanish during reversal, but does fluctuate in magnitude and rotate through zero inclination. During the reversal period of thousands of years, the distribution of magnetic dipole strengths of a bacterial population in a given locale could shift in response to decreasing and increasing field conditions, and the relative numbers of South- and North-seeking cells in the population could change in response to changes in the magnetic inclination.

\section{R. B. FrankeL}

Francis Bitter National Magnet

Laboratory, Massachusetts Institute of

Technology, Cambridge 02139

R. P. BLAKEMORE

Department of Microbiology,

University of New Hampshire,

Durham 03824

F. F. Torres de Araujo

Department of Physics,

Universidade Federal do Ceará,

Fortaleza, Ceará, Brazil

D. M. S. ESQuivel

J. DANON

Centro Brasileiro de Pesquisas Fisicas,

Rio de Janeiro, Brazil

\section{References and Notes}

1. R. P. Blakemore, Science 190, 377 (1975).

2. A. J. Kalmijn and R. P. Blakemore, Animal Migration, Navigation and Homing, $\mathrm{K}$ Schmidt-Koenig and W. T. Keeton, Eds. (Springer-Verlag, New York, 1978), p. 344.

3. T. T. Moench and W. A. Konetzka, Arch. Microbiol. 119, 203 (1978).

4. R. P. Blakemore, R. B. Frankel, A. J. Kalmijn, Nature (London) 286, 384 (1980).

5. J. L. Kirschvink, J. Exp. Biol. 86, 345 (1980).

6. R. B. Frankel, R. P. Blakemore, R. S. Wolfe, Science 203, 1355 (1979).

7. R. B. Frankel and R. P. Blakemore, J. Magn. Magn. Mater. 15-18, 1562 (1980).

8. World Data Center, National Oceanic and Atmospheric Administration, Boulder, Colo.

9. F. S. da Cruz and W. de Sousa, private communication.

10. D. L. Balkwill, D. Maratea, R. P. Blakemore, $J$ Bacteriol. 141, 1399 (1980).

11. C. R. Denham, R. P. Blakemore, R. B. Frankel, IEEE Trans. Magn. Magn-16, 1006 (1980)

12. K. M. Towe and T. T. Moench, Earth Planet. Sci. Lett. 52, 213 (1981).

13. R. P. Blakemore et al., unpublished.

14. E. M. Purcell, Am J. Phys. 45, 3 (1977).

15. R. P. Blakemore, D. Maratea, R. S. Wolfe, $J$ Bacteriol. 140, 720 (1979).

16. B. D. Teague, M. K. Gilson, A. J. Kalmijn, Biol. Bull. (Woods Hole, Mass.) 157, 399 (1979); A. J. Kalmijn, in preparation.

17. A. Cox, Rev. Geophys. Sp. Sci. 13, 35 (1975).

18. We thank A. J. Kalmijn and V Wescott for discussions: H Maia $H$ Lins de Barros, $W$ discussions; H. Maia, H. Lins de Barros, W. O'Brien, R. Caplan, and H. Stram for experimental assistance; and F. da Cruz and $W_{\text {. de }}$ Sousa for electron microscopy. Supported by the U.S. Office of Naval Research, the Organization of American States, and the Conselho Nacional de Desenvolvimento Cientifico e Technologico of Brazil, and NSF grant PCM7922224 (to R.P.B.). The Francis Bitter National Magnet Laboratory is supported by NSF. 